Bangladesh J. Bot. 49(2): 205-213, 2020 (June)

\title{
GROWTH AND PHYSIOLOGICAL RESPONSE OF SELECTED CLONES OF RUBBER GROWN UNDER DIFFERENT WATER FREQUENCIES
}

\author{
Rohaizad Mislan, Z Sulaiman ${ }^{*}$, WD Noordin, SNA Abdullah ${ }^{1}$, MR Ismail, \\ A Selamat, Mya Samad ${ }^{2}$, AB Puteh and MA Salisu \\ Department of Crop Science, Faculty of Agriculture, Universiti Putra Malaysia, \\ 43400 UPM Serdang, Selangor, Malaysia
}

Keywords: Hevea brasiliensis, Latex timber clones, Watering frequencies, Growth, Physiological responses

\begin{abstract}
Effects of water frequencies on growth and physiological response of different clones of rubber were investigated. Different clones of rubber were screened with different watering frequencies as everyday watering (EW), every 2 days (E2D), every 3 days (E3D), every 5 days (E5D), and every 7 days (E7D). The treatments EW and E2D were found to be suitable for all the five clones for increasing as shown in plant height. A similar result was also found for plant biomass after 4 and 8 months of treatments. Noticeably, watering had a pronounced positive effect on clone RRIM 3001 and greatly increased vigorous growth as shown in its highest height, largest girth circumference and relative growth rate after 8 months of different watering frequencies. This clone equally showed superior performance with a significantly higher total plant biomass after 4 and 8 months of watering frequencies compared to the other four clones. The result could be used in water management and the clone RRIM 3001 could be suitable for rubber production at the nursery stage and replanting exercise in rubber plantations.
\end{abstract}

\section{Introduction}

Latex timber clones (LTCs) is known for its adaptation to Malaysian climate, but it may be affected by water stress condition because of the northern and western coast of Peninsular Malaysian experience dry conditions from May to September, leading to drought conditions in many areas. This condition is believed to be the effect of the global warming phenomenon that shifted the rainfall patterns over different regions of Malaysia. Low water availability is the major environmental factor limiting growth, development, and the agricultural production of plants worldwide (Silva et al. 2013). An estimated one-third of the world's terrestrial area suffers from water stress, which is predicted to increase owing to global warming, enhancing the reduction in crop production in many key production regions (Tack et al. 2015).

Physiologically, several plant processes are negatively affected by water stress. These effects occur through osmotic stress and different biochemical responses in plants such as cell turgidity, stomatal conductance, transpiration, photosynthesis, respiration, antioxidant activity, and light absorption and capture, resulting in reduced crop production (Velázquez-Márquez et al. 2015). According to Chaves et al. (2002), the effects of low water availability on plant physiological processes are influenced by both the intensity and duration of the environmental stress as well as the genetic capacity of the genotype/species to cope with stress. The growth and development of plant depend on continued cell division and on progressive tissue differentiation and expansion until the characteristic form of the plant morphologies and physical structure of the plants developed. Plant growth and development involve plant water uptakes and translocations within the plant body.

*Author for correspondence: <zulkefly@upm.edu.my>. ${ }^{1}$ Institute of Plantation Studies, Universiti Putra Malaysia, 43400 UPM Serdang, Selangor, Malaysia. ${ }^{2}$ Department of Land Management, Faculty of Agriculture, Universiti Putra Malaysia, 43400 UPM Serdang, Selangor, Malaysia. 
Plants growing in soil at field capacity soil moisture level could be under water stress.This phenomenon exists because plant water stress develops as a result of the excessive rate of water loss and an inadequate rate of absorption of water. In areas where water is limited and due to a reduction in land designated for rubber planting areas, the effects of water stress on the physiological and growth of rubber LTCs are of particular interest for the establishment of LTCs of rubber clone. To date, there are limited data which would allow the prediction of growth responses of this crop as a result of varying levels of water stress. Thus, the present study was carried out to evaluate the effect of water stress on rubber focusing mainly on responses to water frequencies under stimulated water stress environments.

\section{Materials and Methods}

The experiment was conducted at Field 2, University Putra Malaysia under a rain shelter house with the arched roof covered by ultraviolet (UV)-resistant clear or transparent polyethene (PE) film, $0.1 \mathrm{~mm}$ thick. Five LTCs seedlings (budded plant) aged 4 months (3 leaves whorls) used are LTCs RRIM 3001, 2025, 2001, 928 and PB350. The seedlings were grown in polybag size $20 \times 20$ inches and filled with $35 \mathrm{~kg}$ Munchong Series (Typic Hapludox/Haplic Ferralsol) soil. The soil moisture content was determined using water retention method (Teh and Jamal 2006) with the formula:

Wet basis $(\%)=\left[\mathrm{W}_{\mathrm{d}}-\mathrm{D}_{\mathrm{d}}(\mathrm{g}) / \mathrm{W}_{\mathrm{d}}-\mathrm{D}(\mathrm{g})\right] \times 100$

Dry basis $(\%)=\left[\mathrm{W}_{\mathrm{d}}-\mathrm{D}_{\mathrm{d}}(\mathrm{g}) / \mathrm{D}_{\mathrm{d}}-\mathrm{D}(\mathrm{g})\right] \times 100$ or $\left[\mathrm{W}_{\mathrm{b}}(\%) / 100-\mathrm{W}_{\mathrm{b}}(\%)\right] \times 100$

Bulk density $=\left[D_{\mathrm{w}}(\mathrm{g}) / \mathrm{R}_{\mathrm{v}}\left(\mathrm{cm}^{3}\right)\right.$

$\%$ moisture content $(\mathrm{w} / \mathrm{w})=$ Dry basis $(\%)$

$\%$ moisture content $(\mathrm{v} / \mathrm{v})=$ Dry basis $(\%) \times \mathrm{Bd}\left(\mathrm{gm} / \mathrm{cm}^{3}\right)$

where, $\mathrm{W}_{\mathrm{d}}=$ Wet weight of the soil with dish, $\mathrm{D}_{\mathrm{d}}=$ Dry weight of the soil with dish, $\mathrm{D}=$ Dish weight, $\mathrm{W}_{\mathrm{b}}=$ Wet basis moisture content, $\mathrm{B}_{\mathrm{d}}=$ Bulk density, $\mathrm{D}_{\mathrm{w}}=$ Dry weight of the soil $\mathrm{R}_{\mathrm{v}}=$ Ring volume.

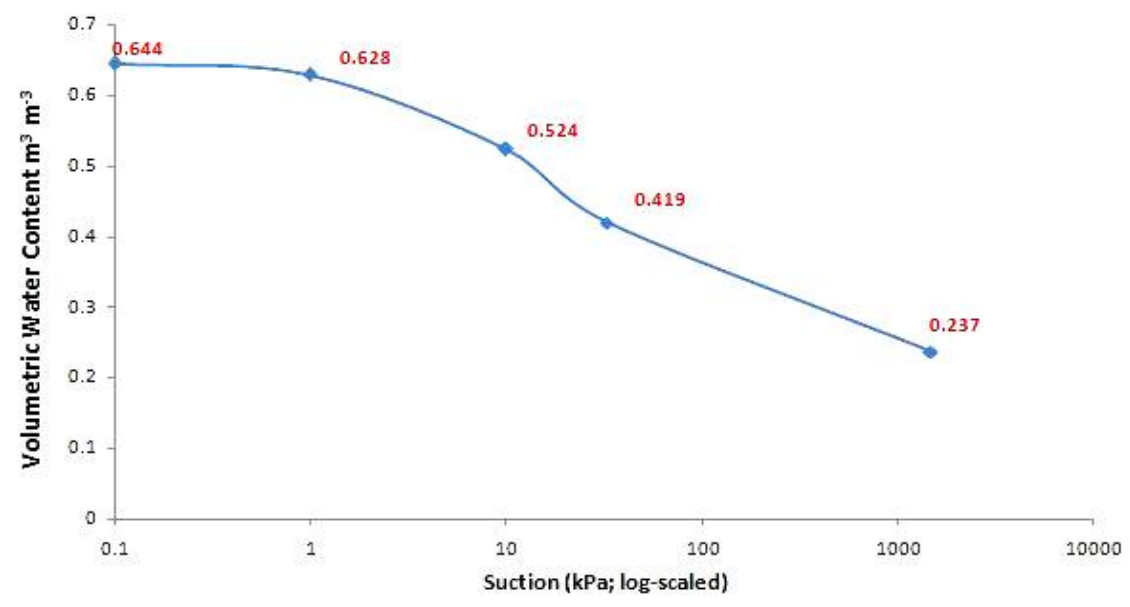

Fig. 1. Munchong series (Typic Hapludox/ Haplic Ferralsol) soil water retention curve.

Average dry basis (\%) different pressure chamber suctions was plotted to the log scale chart with the volumetric water content $\left(\mathrm{m}^{3} \mathrm{~m}^{-3}\right)$ on the $\mathrm{y}$-axis and the soil suction on the $\mathrm{x}$-axis at different pressure: 0, 1, 10,33 and $1500 \mathrm{kPa}$ (Fig. 1). Five watering frequencies were adopted as 
treatments $(\mathrm{EW}=$ Everyday watering, E2D $=$ Every 2 days watering, E3D = Every 3 days watering, E5D = Every 5 days watering and E7D = Every 7 days watering) and subsequent recovery (Field capacity total volumetric water content $0.419 \mathrm{~m}^{3} \mathrm{~m}^{-3}$ ). The soil moisture was measured using portable soil moisture meter $\mathrm{ECH}_{2} \mathrm{O}$ echo probe with echo check meter. The experiment was carried out until the seedlings reach the age of 12 months. Soil water requirement was calculated base on volumetric water content as follows:

[(Field capacity $\times$ height of soil $)-($ Current soil moisture $\times$ height of soil $)] \times[$ Area $]$

Morphological data such as vegetative growth consisting of plant height and girth circumference were measured in $\mathrm{cm}$ at $15 \mathrm{~cm}$ from the budding point. Plants were harvested at 4 and 8 months after treatments (MAT). The dry weight of plant stem, leaf and root was determined after drying to a constant weight at $80^{\circ} \mathrm{C}$ oven dried for $72 \mathrm{hrs}$ and the plant biomass was determined using a weighing scale. Relative growth rates were calculated using the formula described by Gardner et al. (1990). Relative water content (RWC) was taken and calculated as described by González and González (2001).

\section{Results and Discussion}

Result of interaction between clone and watering frequencies are presented in Table 1 . There was significant difference $(\mathrm{p} \leq 0.01)$ at 4 and 6 (MAT) of the plant growth as shown in plant height increment. RRIM 2025 produced the greatest plant height increment with everyday watering (EW) and every 2 days watering (E2D) compared to water stress treatment of E3D, E5D and E7D. This may be due to high photosynthesis rate and root moisture absorption rates (Mokhatar et al. 2011). Clone RRIM 3001 with EW treatment had higher plant height increment compared to the E2D treatment but similar result was found between the treatment E2D and E3D but higher than the treatments E5D and E7D $(87.72>82.97 \mathrm{~cm}=82.87>77.7>71.9 \mathrm{~cm}$, respectively), at 6 (MAT). The result also showed that, RRIM 928 and RRIM 3001 with the treatment E5D had the highest plant height increment compared to the RRIM 2025, PB 350 and RRIM 2001. According to Nobel (2009) the actual water content in plant cells is changed by changing the types of cells and physiological conditions of plants and water uptake by plants is essential for cell expansion process which is an important element in the process of plant growth.

Result of interaction between clone and watering frequencies are presented in Table 2. There was significant difference between clones and watering interaction $(\mathrm{p} \leq 0.01)$ on plant girth circumference at 2 (MAT). The result found that at 2 (MAT) imposed, RRIM 3001 with EW had bigger plant girth than the treatment E2D and E5D. Also at 8 (MAT), clone RRIM 3001 showed largest plant girth than RRIM 2005, RRIM 2001, RRIM 928 and PB 350. This could have been due to the quality of the clone among the latest clones with better interaction with soil and water treatment. A similar result was reported by Salisu et al. (2013). Noticeably, RRIM 928 with the treatment EW had bigger plant girth than the treatment E7D. This indicated that the clone could perform optimally when well watered EW (Rodrigues et al. 1995).

There was a significant interaction between various $H$. brasiliensis LTCs affected with different watering frequencies at 8 (MAT) $(\mathrm{p} \leq 0.001)$. The highest total plant biomass was recorded from RRIM 3001 with everyday watering (EW) and every 2 days watering (E2D) compared to other clones as shown in Table 3 . The critical time for the recovery of photosynthesis was recognized and this aided plant growth and another biomass yield as shown in the total plant biomass of the clone RRIM 3001. Highest total plant biomass reported at 8 (MAT) was imposed. This was obvious in RRIM 3001 with everyday watering (EW) and every 2 days watering (E2D) compared to other clones. This was similar to the result of a study conducted by Pandey et al. (2000). 
Table 1. Effect of watering frequencies and rubber clones on plant height increment.

\begin{tabular}{|c|c|c|c|c|c|c|}
\hline \multirow{2}{*}{ Clones } & \multirow{2}{*}{$\begin{array}{l}\text { Watering } \\
\text { frequencies }\end{array}$} & \multicolumn{5}{|c|}{ Plant height increments $(\mathrm{cm})$} \\
\hline & & 2 Months & 4 Months & \multirow{2}{*}{$\begin{array}{l}6 \text { Months } \\
87.72 \pm 2.45 \mathrm{a}\end{array}$} & \multicolumn{2}{|c|}{8 Months } \\
\hline \multirow{5}{*}{$\begin{array}{c}\text { RRIM } \\
3001\end{array}$} & EW & $24.12 \pm 2.78 \mathrm{abc}$ & $51.22 \pm 0.65 \mathrm{abc}$ & & \multicolumn{2}{|c|}{$119.57 \pm 2.58 \mathrm{ab}$} \\
\hline & E2D & $23.85 \pm 2.59 \mathrm{abc}$ & $50.72 \pm 0.89 \mathrm{abcd}$ & $82.97 \pm 1.66 \mathrm{bcd}$ & \multicolumn{2}{|c|}{$118.30 \pm 3.54 \mathrm{abcd}$} \\
\hline & E3D & $17.50 \pm 2.50 \mathrm{defgh}$ & $50.25 \pm 0.48 \mathrm{abcd}$ & $82.87 \pm 0.85 \mathrm{bcd}$ & \multicolumn{2}{|c|}{$115.55 \pm 0.61 \mathrm{bcdefg}$} \\
\hline & E5D & $11.80 \pm 1.70 \mathrm{hijk}$ & $43.67 \pm 0.78$ fgh & $77.77 \pm 1.87 \mathrm{efg}$ & \multicolumn{2}{|c|}{$110.45 \pm 1.46 \mathrm{gh}$} \\
\hline & E7D & $11.80 \pm 1.79$ hijk & $44.77 \pm 1.08 \mathrm{fg}$ & $71.95 \pm 1.76 \mathrm{hij}$ & \multicolumn{2}{|c|}{$111.00 \pm 0.93 \mathrm{gh}$} \\
\hline \multirow{5}{*}{$\begin{array}{l}\text { RRIM } \\
2025\end{array}$} & EW & $26.40 \pm 2.34 \mathrm{a}$ & $53.77 \pm 1.15 \mathrm{a}$ & $86.75 \pm 0.98 \mathrm{ab}$ & \multicolumn{2}{|c|}{$117.50 \pm 1.11 \mathrm{abcde}$} \\
\hline & E2D & $21.20 \pm 2.73 \mathrm{abcdef}$ & $51.85 \pm 1.97 \mathrm{ab}$ & $84.97 \pm 1.61 \mathrm{abc}$ & \multicolumn{2}{|c|}{$116.82 \pm 1.14 \mathrm{abcdef}$} \\
\hline & E3D & $15.00 \pm 2.88$ fghij & $45.82 \pm 1.88 \mathrm{efg}$ & $79.22 \pm 1.67 \mathrm{def}$ & \multicolumn{2}{|c|}{$111.50 \pm 1.08 \mathrm{fgh}$} \\
\hline & E5D & $15.62 \pm 1.51$ efghi & $44.05 \pm 1.21 \mathrm{fgh}$ & $71.00 \pm 1.23 \mathrm{ij}$ & \multicolumn{2}{|c|}{$108.27 \pm 1.89 \mathrm{hi}$} \\
\hline & E7D & $13.22 \pm 1.54$ ghijk & $47.22 \pm 2.29 \mathrm{cdef}$ & $76.27 \pm 1.67 \mathrm{fgh}$ & \multicolumn{2}{|c|}{$108.37 \pm 1.01 \mathrm{hi}$} \\
\hline \multirow{5}{*}{$\begin{array}{l}\text { RRIM } \\
2001\end{array}$} & EW & $19.67 \pm 1.88$ bcdef & $52.92 \pm 1.34 \mathrm{ab}$ & $83.10 \pm 1.00 \mathrm{bcd}$ & \multicolumn{2}{|c|}{$115.42 \pm 0.67 \mathrm{bcdefg}$} \\
\hline & E2D & $24.62 \pm 0.79 \mathrm{ab}$ & $52.40 \pm 0.88 \mathrm{ab}$ & $86.32 \pm 0.77 \mathrm{ab}$ & \multicolumn{2}{|c|}{$118.62 \pm 3.61 \mathrm{abcd}$} \\
\hline & E3D & $16.27 \pm 2.17$ efghi & $45.27 \pm 1.35 \mathrm{efg}$ & $77.70 \pm 1.97 \mathrm{efg}$ & \multicolumn{2}{|c|}{$111.80 \pm 1.62 \mathrm{efgh}$} \\
\hline & E5D & $15.70 \pm 0.95$ efghi & $46.90 \pm 0.49 \mathrm{def}$ & $73.87 \pm 0.46 \mathrm{ghi}$ & \multicolumn{2}{|c|}{$111.45 \pm 1.13 \mathrm{fgh}$} \\
\hline & E7D & $9.00 \pm 0.80 \mathrm{jk}$ & $40.15 \pm 1.24 \mathrm{hi}$ & $68.77 \pm 1.78 \mathrm{j}$ & \multicolumn{2}{|c|}{$103.70 \pm 0.74 \mathrm{ij}$} \\
\hline \multirow{5}{*}{$\begin{array}{l}\text { RRIM } \\
928\end{array}$} & EW & $25.45 \pm 4.22 \mathrm{ab}$ & $51.77 \pm 2.23 \mathrm{abcd}$ & $84.25 \pm 2.61 \mathrm{abc}$ & \multicolumn{2}{|c|}{$118.97 \pm 1.92 \mathrm{abcd}$} \\
\hline & E2D & $21.62 \pm 1.80 \mathrm{abcde}$ & $53.55 \pm 1.63 \mathrm{a}$ & $84.60 \pm 0.34 a b c$ & \multicolumn{2}{|c|}{$121.47 \pm 3.42 \mathrm{a}$} \\
\hline & E3D & $20.55 \pm 0.51 \mathrm{abcdef}$ & $46.92 \pm 1.18 \mathrm{def}$ & $79.22 \pm 0.68 \mathrm{def}$ & \multicolumn{2}{|c|}{$114.77 \pm 1.14$ bcdefg } \\
\hline & E5D & $15.62 \pm 1.80$ efghi & $47.12 \pm 1.77 \mathrm{def}$ & $78.05 \pm 1.97 \mathrm{efg}$ & 113. & $2 \pm 2.63$ defgh \\
\hline & E7D & $8.57 \pm 1.58 \mathrm{k}$ & $38.97 \pm 0.76 \mathrm{i}$ & $69.20 \pm 1.31 \mathrm{j}$ & 99.5 & $\pm 2.17 \mathrm{j}$ \\
\hline & EW & $23.55 \pm 3.53 \mathrm{abcd}$ & $52.05 \pm 0.95 \mathrm{ab}$ & $84.30 \pm 0.70 \mathrm{abc}$ & 118. & $2 \pm 1.65 \mathrm{abcd}$ \\
\hline & E2D & $23.95 \pm 1.55 \mathrm{abc}$ & $51.67 \pm 1.82 \mathrm{ab}$ & $84.10 \pm 1.41 \mathrm{abc}$ & 119. & $0 \pm 2.38 \mathrm{abc}$ \\
\hline PB 350 & E3D & $18.30 \pm 3.19 \mathrm{cdefg}$ & $48.97 \pm 1.91$ bcde & $81.45 \pm 2.48 \mathrm{cde}$ & 113. & $0 \pm 2.79$ cdefgh \\
\hline & E5D & $8.87 \pm 2.03 \mathrm{jk}$ & $42.25 \pm 0.60$ ghi & $70.00 \pm 0.83 \mathrm{ij}$ & 108. & $5 \pm 2.69 \mathrm{hi}$ \\
\hline & E7D & $10.10 \pm 0.81 \mathrm{ijk}$ & $42.35 \pm 2.32 \mathrm{ghi}$ & $69.97 \pm 1.15 \mathrm{ij}$ & 103. & $7 \pm 1.42 \mathrm{ij}$ \\
\hline Source & & & F value & Ipprox. $\operatorname{Pr}>$ F) & & \\
\hline & & 2 Months & Months & 6 Months & & 8 Months \\
\hline Clones & & $0.46 \quad(0.7668)$ & $50 \quad(0.7322)$ & $2.81(0.0318$ & & $1.54(0.1985)$ \\
\hline Watering & & $34.80(<0.0001)$ & $4.02(<0.0001)$ & $81.83(<0.0$ & $001)$ & $37.16(<0.0001)$ \\
\hline Clones $x$ & watering & $1.27 \quad(0.2430)$ & $54(0.0037)$ & $2.75(0.0018$ & & $1.60(0.0915)$ \\
\hline
\end{tabular}

Mean \pm SE $(n=4)$ and F-test non-significant or significant at $p<0.05,0.01$ or 0.001 , respectively. Values followed by the same letter within each vertical column $(a, b, c)$ are not significantly different with the least significant level of 5\% (LSD). 
Table 2. Effect of watering frequencies and rubber clones on plant girth circumference.

\begin{tabular}{|c|c|c|c|c|c|}
\hline \multirow[t]{2}{*}{ Clones } & \multirow{2}{*}{$\begin{array}{l}\text { Watering } \\
\text { frequencies }\end{array}$} & \multicolumn{4}{|c|}{ Plant Girth Circumference $(\mathrm{cm})$} \\
\hline & & 2 Months & 4 Months & 6 Months & 8 Months \\
\hline \multirow{5}{*}{$\begin{array}{l}\text { RRIM } \\
3001\end{array}$} & EW & $2.40 \pm 0.07 \mathrm{a}$ & $4.17 \pm 0.11 \mathrm{abc}$ & $6.82 \pm 0.34 \mathrm{ab}$ & $8.37 \pm 0.11 \mathrm{ab}$ \\
\hline & E2D & $2.00 \pm 0.36 \mathrm{bcd}$ & $4.27 \pm 0.16 \mathrm{ab}$ & $6.97 \pm 0.23 \mathrm{a}$ & $8.75 \pm 0.06 a$ \\
\hline & E3D & $1.62 \pm 0.11$ defghij & $3.50 \pm 0.21 \mathrm{e}$ & $4.57 \pm 0.23 \mathrm{~d}$ & $7.30 \pm 0.17 \mathrm{def}$ \\
\hline & E5D & $1.40 \pm 0.14 \mathrm{hijk}$ & $3.37 \pm 0.22 \mathrm{ef}$ & $4.20 \pm 0.26 \mathrm{de}$ & $6.77 \pm 0.17 \mathrm{fgh}$ \\
\hline & E7D & $1.27 \pm 0.17 \mathrm{jk}$ & $2.35 \pm 0.15 \mathrm{i}$ & $3.32 \pm 0.16 f g$ & $6.55 \pm 0.18 \mathrm{ij}$ \\
\hline \multirow{5}{*}{$\begin{array}{l}\text { RRIM } \\
2025\end{array}$} & EW & $1.95 \pm 0.24 \mathrm{cde}$ & $4.15 \pm 0.22 \mathrm{abc}$ & $6.82 \pm 0.38 \mathrm{ab}$ & $8.15 \pm 0.15 b c$ \\
\hline & E2D & $1.80 \pm 0.07 \mathrm{cdefg}$ & $4.15 \pm 0.11 \mathrm{abc}$ & $6.27 \pm 0.36 \mathrm{bc}$ & $7.95 \pm 0.15 b c$ \\
\hline & E3D & $1.82 \pm 0.13 \mathrm{cdefg}$ & $3.82 \pm 0.23$ bcde & $4.40 \pm 0.26 \mathrm{de}$ & $6.85 \pm 0.32 \mathrm{efgh}$ \\
\hline & E5D & $1.57 \pm 0.08$ efghij & $3.52 \pm 0.17 \mathrm{e}$ & $4.35 \pm 0.09 \mathrm{de}$ & $6.82 \pm 0.13$ efgh \\
\hline & E7D & $1.47 \pm 0.10$ ghijk & $2.40 \pm 0.08 \mathrm{hi}$ & $3.45 \pm 0.08 \mathrm{fg}$ & $6.17 \pm 0.18 \mathrm{hi}$ \\
\hline \multirow{5}{*}{$\begin{array}{l}\text { RRIM } \\
2001\end{array}$} & EW & $1.75 \pm 0.15$ cdefgh & $4.15 \pm 0.05 \mathrm{abc}$ & $6.95 \pm 0.39 \mathrm{a}$ & $8.17 \pm 0.23 b$ \\
\hline & E2D & $2.02 \pm 0.02 \mathrm{abc}$ & $4.45 \pm 0.16 a$ & $6.02 \pm 0.04 c$ & $8.02 \pm 0.13 b c$ \\
\hline & E3D & $1.77 \pm 0.07$ cdefgh & $3.55 \pm 0.05 \mathrm{e}$ & $4.67 \pm 0.04 \mathrm{~d}$ & $6.75 \pm 0.19 \mathrm{fgh}$ \\
\hline & E5D & $1.65 \pm 0.15$ cdefghij & $3.52 \pm 0.17 \mathrm{e}$ & $4.10 \pm 0.16 \mathrm{de}$ & $6.50 \pm 0.23 \mathrm{ghi}$ \\
\hline & E7D & $1.57 \pm 0.10$ efghij & $2.35 \pm 0.14 \mathrm{i}$ & $3.35 \pm 0.11 \mathrm{fg}$ & $5.72 \pm 0.18 \mathrm{jk}$ \\
\hline \multirow[t]{5}{*}{ RRIM 928} & EW & $1.87 \pm 0.10 \mathrm{cdef}$ & $4.17 \pm 0.08 \mathrm{abc}$ & $6.02 \pm 0.21 \mathrm{c}$ & $7.60 \pm 0.31 \mathrm{~cd}$ \\
\hline & E2D & $1.62 \pm 0.13$ defghij & $4.02 \pm 0.13 \mathrm{abcd}$ & $5.90 \pm 0.04 \mathrm{c}$ & $7.37 \pm 0.07 \mathrm{de}$ \\
\hline & E3D & $1.87 \pm 0.04 \mathrm{cdef}$ & $3.75 \pm 0.15 \mathrm{cdef}$ & $4.55 \pm 0.02 d$ & $6.97 \pm 0.13 \mathrm{efg}$ \\
\hline & E5D & $1.67 \pm 0.08$ cdefghi & $3.02 \pm 0.16 f g$ & $4.15 \pm 0.12 \mathrm{de}$ & $6.37 \pm 0.19 \mathrm{hi}$ \\
\hline & E7D & $1.47 \pm 0.14$ ghijk & $2.20 \pm 0.17 \mathrm{i}$ & $3.05 \pm 0.19 \mathrm{~g}$ & $5.32 \pm 0.17 \mathrm{jk}$ \\
\hline \multirow[t]{5}{*}{ PB 350} & EW & $2.37 \pm 0.075 \mathrm{ab}$ & $4.20 \pm 0.14 \mathrm{abc}$ & $6.97 \pm 0.38 \mathrm{a}$ & $8.05 \pm 0.25 b c$ \\
\hline & E2D & $1.75 \pm 0.05 \mathrm{cdefgh}$ & $4.25 \pm 0.20 \mathrm{ab}$ & $6.50 \pm 0.21 \mathrm{abc} 7$ & $7.95 \pm 0.15 b c$ \\
\hline & E3D & $1.32 \pm 0.13 \mathrm{ijk}$ & $3.62 \pm 0.13 \mathrm{de}$ & $4.62 \pm 0.13 d$ & $7.05 \pm 0.22 \mathrm{defg}$ \\
\hline & E5D & $1.50 \pm 0.12$ fghijk & $2.82 \pm 0.07 \mathrm{gh}$ & $3.82 \pm 0.11 \mathrm{ef}$ & $6.55 \pm 0.18 \mathrm{ghi}$ \\
\hline & E7D & $1.12 \pm 0.11 \mathrm{k}$ & $2.05 \pm 0.20 \mathrm{i}$ & $3.15 \pm 0.21 \mathrm{~g}$ & $6.05 \pm 0.32 \mathrm{ij}$ \\
\hline \multirow{2}{*}{\multicolumn{2}{|c|}{ Source }} & \multicolumn{4}{|c|}{ F value (Approx. $\operatorname{Pr}>$ F) } \\
\hline & & 2 Months & 4 Months & 6 Months & 8 Months \\
\hline \multicolumn{2}{|l|}{ Clones } & $0.78(0.5406)$ & $1.92(0.1158)$ & $2.72(0.0359)$ & $11.31(<.0001)$ \\
\hline \multicolumn{2}{|l|}{ Watering } & $17.67(<0.0001)$ & $125.27(<0.0001)$ & $222.01(<0.0001)$ & ) $107.36(<0.0001)$ \\
\hline \multicolumn{2}{|c|}{ Clones $\times$ watering } & $2.54(0.0038)$ & $1.11(0.3595)$ & $1.42(0.1593)$ & $1.18 \quad(0.3058)$ \\
\hline
\end{tabular}

Mean \pm SE $(n=4)$ and F-test non-significant or significant at, $p<0.05,0.01$ or 0.001 , respectively. Values followed by the same letter within each vertical column $(a, b, c)$ are not significantly different with the least significant level of 5\% (LSD). 
Table 3. Effect of watering frequencies on total plant biomass of selected rubber clones.

\begin{tabular}{|c|c|c|c|}
\hline \multirow{2}{*}{ Clones } & \multirow{2}{*}{$\begin{array}{l}\text { Watering } \\
\text { frequencies }\end{array}$} & \multicolumn{2}{|c|}{ Total biomass (g/tree) } \\
\hline & & 4 Months & 8 Months \\
\hline \multirow{5}{*}{ RRIM 3001} & EW & $820.25 \pm 6.97 \mathrm{a}$ & $2539.50 \pm 29.35 \mathrm{a}$ \\
\hline & E2D & $837.50 \pm 29.56 a$ & $2613.50 \pm 92.72 \mathrm{a}$ \\
\hline & E3D & $725.25 \pm 10.28 b$ & $1892.50 \pm 114.69 \mathrm{ef}$ \\
\hline & E5D & $709.25 \pm 6.45 b$ & $1518.50 \pm 23.21 \mathrm{ij}$ \\
\hline & E7D & $664.50 \pm 2.63 \mathrm{de}$ & $1513.00 \pm 31.11 \mathrm{ij}$ \\
\hline \multirow{5}{*}{ RRIM 2025} & EW & $809.00 \pm 5.73 a$ & $2330.75 \pm 61.30 \mathrm{~b}$ \\
\hline & E2D & $807.50 \pm 9.74 a$ & $2346.25 \pm 41.56 b$ \\
\hline & E3D & $703.00 \pm 8.12 b c$ & $1614.00 \pm 73.34 \mathrm{hi}$ \\
\hline & E5D & $685.25 \pm 7.19 \mathrm{bcd}$ & $1501.25 \pm 26.29 \mathrm{ij}$ \\
\hline & E7D & $640.50 \pm 12.02 \mathrm{e}$ & $1475.50 \pm 26.62 \mathrm{ij}$ \\
\hline \multirow{5}{*}{ RRIM 2001} & EW & $791.00 \pm 1.47 \mathrm{a}$ & $2072.75 \pm 67.45 \mathrm{~cd}$ \\
\hline & E2D & $802.00 \pm 8.28 \mathrm{a}$ & $2234.75 \pm 46.71 b c$ \\
\hline & E3D & $715.75 \pm 4.15 b c$ & $1593.75 \pm 49.06 \mathrm{hi}$ \\
\hline & E5D & $681.75 \pm 3.92 \mathrm{cde}$ & $1522.00 \pm 47.52 \mathrm{ij}$ \\
\hline & E7D & $639.75 \pm 7.46 \mathrm{de}$ & $1445.00 \pm 81.52 \mathrm{ij}$ \\
\hline \multirow{5}{*}{ RRIM 928} & EW & $805.75 \pm 18.31 \mathrm{a}$ & $2038.50 \pm 94.76 \mathrm{de}$ \\
\hline & E2D & $796.00 \pm 2.16 \mathrm{a}$ & $2054.50 \pm 58.45 \mathrm{de}$ \\
\hline & E3D & $692.75 \pm 6.62 \mathrm{cde}$ & $1699.00 \pm 81.43 \mathrm{gh}$ \\
\hline & E5D & $691.75 \pm 3.14 \mathrm{~cd}$ & $1494.75 \pm 23.32 \mathrm{ij}$ \\
\hline & E7D & $650.75 \pm 2.78 \mathrm{de}$ & $1396.25 \pm 52.41 \mathrm{j}$ \\
\hline \multirow{5}{*}{ PB 350} & EW & $824.50 \pm 4.09 \mathrm{a}$ & $2286.00 \pm 18.76 b$ \\
\hline & E2D & $805.25 \pm 8.48 \mathrm{a}$ & $2274.50 \pm 70.57 b$ \\
\hline & E3D & $699.00 \pm 21.67 \mathrm{cde}$ & $1835.00 \pm 46.60 f g$ \\
\hline & E5D & $674.00 \pm 17.36 \mathrm{de}$ & $1535.25 \pm 37.98 \mathrm{hij}$ \\
\hline & E7D & $651.25 \pm 4.68 \mathrm{cde}$ & $1422.75 \pm 58.83 \mathrm{j}$ \\
\hline \multirow[t]{2}{*}{ Source } & & \multicolumn{2}{|c|}{ F value (Approx. $\operatorname{Pr}>F$ ) } \\
\hline & & 4 Months & 8 Months \\
\hline Clones & & $4.63(0.0022)$ & $16.07(<0.0001)$ \\
\hline Watering & & $227.64(<0.0001)$ & $227.07(<0.0001)$ \\
\hline Clones $\times$ Watering & & $0.72(0.7633)$ & $3.12 \quad(0.0005)$ \\
\hline
\end{tabular}

Mean \pm SE $(n=4)$ and F test non-significant or significant at, $p<0.05,0.01$ or 0.001 , respectively. Values followed by the same letter within each vertical column $(a, b, c)$ are not significantly different with the least significant level of 5\% (LSD). 
Effects of watering frequencies with various LTCs on plant relative growth rate (RGR) are presented in Table 4 . There was a significant interaction between clones and watering frequencies on plant relative growth rate ( $\mathrm{p} \leq 0.05)$. The RRIM 3001 recorded the highest mean of PGR $0.2845 \mathrm{~g} / \mathrm{g}$ compared to other clones. Low RGR values also have a low biomass production and highest values of biomass production produce an average RGR (Erice et al. 2010). Watering frequencies had highly significant effects $(\mathrm{p} \leq 0.0001)$ on plant relative water content at 8 (MAT) as shown in Table 5.

Table 4. Effect of watering frequencies on plant relative growth rate $(\mathrm{g} / \mathrm{g})$ on rubber clones.

\begin{tabular}{lccccc}
\hline & \multicolumn{3}{c}{ Relative growth rate $(\mathrm{g} / \mathrm{g})$} \\
\cline { 2 - 6 } Clones & EW & E2D & E3D & E5D & E7D \\
\hline RRIM & $0.2825 \pm 0.0039 \mathrm{ab}$ & $0.2845 \pm 0.0104 \mathrm{a}$ & $0.2383 \pm 0.0159 \mathrm{efg}$ & $0.1903 \pm 0.0046 \mathrm{j}$ & $0.2055 \pm 0.0044 \mathrm{ij}$ \\
3001 & & & & \\
RRIM & $0.2645 \pm 0.0076 \mathrm{abcd}$ & $0.2665 \pm 0.0067 \mathrm{abc}$ & $0.2073 \pm 0.0099 \mathrm{hij}$ & $0.1960 \pm 0.0041 \mathrm{j}$ & $0.2085 \pm 0.0079 \mathrm{hij}$ \\
2025 & & & & \\
RRIM & $0.2405 \pm 0.0078 \mathrm{defg}$ & $0.2563 \pm 0.0065 \mathrm{cdef}$ & $0.1998 \pm 0.0075 \mathrm{ij}$ & $0.2005 \pm 0.0092 \mathrm{ij}$ & $0.2025 \pm 0.0131 \mathrm{ij}$ \\
2001 & & & & \\
RRIM & $0.2318 \pm 0.0117 \mathrm{fgh}$ & $0.2368 \pm 0.0070 \mathrm{efg}$ & $0.2235 \pm 0.0137 \mathrm{ghi}$ & $0.1928 \pm 0.0039 \mathrm{j}$ & $0.1905 \pm 0.0085 \mathrm{j}$ \\
928 & & & & \\
PB 350 & $0.2550 \pm 0.0011 \mathrm{cdef}$ & $0.2595 \pm 0.0087 \mathrm{bcde}$ & $0.2418 \pm 0.0089 \mathrm{defg}$ & $0.2060 \pm 0.0089 \mathrm{ij}$ & $0.1948 \pm 0.0102 \mathrm{j}$ \\
\hline Source & F value (Approx. Pr $>\mathrm{F})$ & & \\
Clones & $6.43 \quad(0.0002)$ & & & \\
Watering & $58.46(<.0001)$ & & & \\
Clones $\times$ watering & $2.15 \quad(0.0148)$ & & \\
\hline
\end{tabular}

Mean \pm SE $(n=4)$ and F-test non-significant or significant at $p<0.05,0.01$ or 0.001 , respectively. Values followed by the same letter within each vertical column $(a, b, c)$ are not significantly different with the least significant level of $5 \%$ (LSD).

Table 5. Effect of watering frequencies on plant relative water content in rubber clones.

\begin{tabular}{|c|c|c|c|c|c|}
\hline \multirow{2}{*}{ Clones } & \multicolumn{5}{|c|}{ Relative water content $(\%)$} \\
\hline & EW & E2D & E3D & E5D & E7D \\
\hline RRIM 3001 & $89.24 \pm 1.31 \mathrm{a}$ & $86.85 \pm 0.68 \mathrm{ab}$ & $84.33 \pm 1.73$ bcdefg & $82.67 \pm 1.56 \mathrm{cdefgh}$ & $81.68 \pm 2.46 \mathrm{efgh}$ \\
\hline RRIM 2025 & $87.42 \pm 0.31 \mathrm{ab}$ & $85.73 \pm 1.32 \mathrm{abcd}$ & $81.45 \pm 0.88$ fgh & $84.58 \pm 0.96 \mathrm{bcdef}$ & $81.39 \pm 1.04$ fgh \\
\hline RRIM 2001 & $86.59 \pm 0.74 \mathrm{ab}$ & $85.61 \pm 2.21 \mathrm{abcd}$ & $82.19 \pm 2.34$ cdefgh & $80.07 \pm 0.83 \mathrm{~h}$ & $80.70 \pm 2.53 \mathrm{gh}$ \\
\hline RRIM 928 & $86.63 \pm 0.45 \mathrm{ab}$ & $86.62 \pm 1.23 \mathrm{ab}$ & $82.58 \pm 1.34$ cdefgh & $82.36 \pm 1.16 \mathrm{cdefgh}$ & $81.94 \pm 0.90$ defgh \\
\hline PB 350 & $85.95 \pm 1.03 \mathrm{abc}$ & $85.46 \pm 1.97$ abcde & $84.94 \pm 1.93$ bcdef & $82.42 \pm 1.06 \mathrm{cdefgh}$ & $81.49 \pm 1.57 \mathrm{fgh}$ \\
\hline \multicolumn{3}{|l|}{ Source } & \multicolumn{3}{|c|}{ F value (Approx. $\operatorname{Pr}>$ F) } \\
\hline \multicolumn{3}{|l|}{ Clones } & $(0.2942)$ & & \\
\hline \multicolumn{3}{|l|}{ Watering } & $16.38 \quad(0.0001)$ & & \\
\hline \multicolumn{3}{|c|}{ Clones $\times$ watering } & 0.8471 & & \\
\hline
\end{tabular}

Mean \pm SE $(n=4)$ and F-test non-significant or significant at, $\mathrm{p}<0.05,0.01$ or 0.001 , respectively. Values followed by the same letter within each vertical column $(a, b, c)$ are not significantly different with the least significant level of 5\% (LSD).

Clones showed significant difference $(\mathrm{p} \leq 0.01)$ on total plant biomass at 4 and 8 (MAT) ( $\mathrm{p} \leq 0.0001$ ), respectively. Merine et al. (2015) mentioned that, watering frequency could have a significant effect on overall total plant biomass including the stem dry weight. Interestingly, Xu 
et al. (2015) observed that plant biomass could increase under drought stress of some plant species. Manzi et al. (2015) observed that, root could survive under long-term water stress due to the activities of the root aerial organs. Availability of nutrient like $\mathrm{N}$ could equally have a significant impact of plant biomass yield noticeable in leaf dry biomass (Ashraf et al. 2017).

The aforesaid results obviously show the effect of watering frequencies at EW and E2D had a profound effect on the plant biomass at 4 and 8 (MAT) and relative growth rate at 8 (MAT). The water frequencies are equally considered suitable for all the clones used in the study. The effect was noticeable on the RRIM 3001 and the effect of the different watering frequencies was profoundly showed on its total plant biomass at 4 and 8 (MAT) of watering frequencies compared to the other four clones. Consequently, the water frequencies EW and E2D and RRIM 3001 could be considered the most suitable for latex timber production and water management in the rubber plantation industry, specially for the replanting exercise.

\section{References}

Ashraf AN, Zulkefly S, Adekunle S and Samad MYA 2017. Growth and biomass yield of oil palm (Elaeis guineensis) seedlings as influenced by different rates of vermicompost. European J. of Engineer Res. and Sci. 2(8): 17-21.

Bassirirad H 2000. Kinetics of nutrient uptake by roots: Responses to global change. The New Phytologist. 147(1):155-169.

Chaves MM, Pereira JS, Maroco J, Rodrigues M, Ricardo CPP, Osório ML, Carvalho I, Faria T and Pinheiro C 2002. How plants cope with water stress in the field: Photosynthesis and growth. Ann. Bot. 89(7): 907-916.

Erice G, Louahlia S, Irigoyen JJ, Sanchez-Diaz M and Avice JC 2010. Biomass partitioning, morphology and water status of four alfalfa genotypes submitted to progressive drought and subsequent recovery. J. Plant Physiol. 167 (2): 114-120.

Gardner FP, Brent and Mitchell RL 1990. Physiology of crop plants. Iowa State Univ. Press, pp. 200-203.

González L and González-Vilar M 2001. Determination of relative water content. In: Handbook of plant ecophysiology techniques. Springer, Dordrecht. pp. 207-212

Manzi M, Lado J, Rodrigo MJ, Zacarías L, Arbona V and Gómez-Cadenas, A 2015. Root ABA accumulation in long-term water-stressed plants is sustained by hormone transport from aerial organs. Plant Cell Physiology 56(12): 2457-2466.

Merine AK, Rodríguez-García E, Alía R, Pando V and Bravo F 2015. Effects of water stress and substrate fertility on the early growth of Acacia senegal and Acacia seyal from Ethiopian Savanna woodlands. Trees 29(2): 593-604.

Mokhatar SJ, Daud NW, and Zamri NM 2011. Evaluation of different water regimes on Hevea brasiliensis grown on haplic ferralsol soil at nursery stage. Inter J. Applied 1(3):28-33

Nobel PS 2009. Physicochemical and Environmental Plant Physiology. Elsevier Academic Press.

Pandey RK, Maranville JW and Chetima MM 2000. Deficit irrigation and nitrogen effects on maize in a Sahelian environment: II. Shoot growth, nitrogen uptake and water extraction. Agric.Water Management 46(1): 15-27.

Rodrigues ML, Pacheco CMA and Chaves MM 1995. Soil-plant water relations, root distribution and biomass partitioning in Lupinus albus L. under drought conditions. J of Experi Bot.46(8): 947-956.

Salisu M, Daud N and Ahmad I 2013. Influence of fertilizer rates and soil series on growth performance of natural rubber ('Hevea brasiliensis') latex timber clones. Austr J. Crop Sci. 7(13): 1998-2004

Tack J, Barkley A and Nalley LL 2015. Effect of warming temperatures on US wheat yields. Nat. Acad. Sci. United States

Teh CBS and Jamal T 2006. Water Retention. Soil Physics Analysis 4: 14-17. 
Velázquez-Márquez S, Conde-Martínez V, Trejo C, Delgado-Alvarado A, Carballo A, Suárez R, Mascorro JO and Trujillo AR 2015. Effects of water deficit on radicle apex elongation and solute accumulation in Zea mays L. Plant Physiol. Bioc. 96: 29-37.

Xu W, Cui K, Xu A, Nie L, Huang J and Peng S 2015. Drought stress condition increases root to shoot ratio via alteration of carbohydrate partitioning and enzymatic activity in rice seedlings. Acta Physio Plantarum 37(2): 2-9.

(Manuscript received on 12 December, 2018; revised on 24 July 2019) 